\title{
2 Chirurgische Behandlung der morbiden Adipositas und metabolischer Störungen
}

\author{
Rudolf Alfred Weiner
}

Die Chirurgie zur Reduktion des krankhaften Übergewichts (Adipositas), auch Adipositaschirurgie (historischer Begriff: bariatrische Chirurgie) genannt, hat aufgrund des Versagens konservativer Therapiekonzepte und der ungebremsten Zunahme in der Prävalenz von Übergewicht und Adipositas weltweit eine wachsende Bedeutung. Durch die weitere Zunahme des Durchschnitts-BMI der Bevölkerung sind auch zukünftig ansteigende Zahlen von Patienten mit krankhafter Adipositas zu erwarten. Es ist wissenschaftlich belegt, dass die Chirurgie der konservativen, nicht-operativen Therapie in Bezug auf langzeitige Gewichtskontrolle (Andersen et al. 1984), Lebensqualität (Weiner et al. 2007) und Verbesserung von Komorbiditäten überlegen ist (Sjöström et al. 2007). Adipositas ist eine chronische Erkrankung, die derzeit nicht kausal zu behandeln ist. Das trifft auch auf den Diabetes mellitus Typ 2 zu, der ebenfalls durch operative Intervention zur Remission gebracht werden kann. Ist die Indikation für eine operative Therapie das metabolische Syndrom, dann spricht man von metabolischer Chirurgie. Prävention ist die wichtigste gesamtgesellschaftliche Aufgabe zur Lösung des Problems.

\subsection{Klassifikation und Komorbiditäten der Adipositas}

Die Schwere der Adipositas wird nach der WHO-Klassifizierung eingeteilt(s. Tab. 1). Dabei wird derzeitder Body-Mass-Index (BMI) als Berechnungsgrundlage verwendet. Eine Veränderung der Klassifikation auf-
Tab. 1 Klassifizierung der Adipositas nach WHO

\begin{tabular}{|l|c|}
\hline Klassifikation & BMI $\left(\mathrm{kg} / \mathrm{m}^{2}\right)$ \\
\hline Normalgewicht & $18-24,9$ \\
\hline Übergewicht & $25-29,9$ \\
\hline Adipositas Grad I & $30-34,9$ \\
\hline Adipositas Grad II & $35-39,9$ \\
\hline Adipositas Grad III (morbide Adipositas) & $>40$ \\
\hline Superadipositas & $>50$ \\
\hline
\end{tabular}

grund der Zunahme von Extremformen und der Risikolage ist in Diskussion. Ein BMI von 25 stellt im Erwachsenenalter das Idealgewicht dar. Jedes Gewicht darüber bezeichnet man definitionsgemäß als Übergewicht. Geschlechtsspezifische und ethnische Unterschiede sind definiert.

$$
\text { BMI = Körpergewicht }(\mathrm{kg}) / \text { Körpergröße }\left(\mathrm{m}^{2}\right)
$$

Die Adipositas ist eng assoziiert mit dem metabolischen Syndrom. Durch Hypertonie, Diabetes mellitus Typ 2, Hypercholesterinämie und Dyslipidämie wird die Entwicklung der Arteriosklerose mit koronarer Herzkrankheit und eine kürzere Lebensdauer 
durch kardiovaskuläre Komplikationen begünstigt. Weiterhin treten mit hoher Inzidenz eine obstruktive Schlafapnoe, Gallenblasenerkrankungen, degenerative Gelenkerkrankungen, und eine Vielzahl von weiteren Erkrankungen auf. Durch ein vermehrtes Auftreten von Karzinomen (Kolon, Gallenblase, Pankreas Leber, Mamma, Endometrium, Ovarien, Zervix, Prostata) wird die Lebenserwartung der Adipösen gegenüber Normalgewichtigen weiter signifikant verkürzt. Eine Gewichtsreduktion verbessert dagegen die Lebenserwartung (Adams et al. 2007; Christou et al. 2004; Sjöström et al. 2007). Allein die Sterblichkeit an Krebserkrankungen wurde im 5-Jahres-Intervall um 60\% verringert (Adams et al. 2007).

\subsection{Epidemiologie}

Die Inzidenz der morbiden Adipositas hat in den vergangenen Jahren nicht nur in der westlichen Welt stetig zugenommen (WHO 200o). In Deutschland kam es in den letzten Jahren zu einer erheblichen Zunahme der Adipositasprävalenz sowohl bei Erwachsenen als auch bei Kindern. Repräsentative Daten für die Auftretenshäufigkeit der Adipositas wurden in vier Gesundheitssurveys zwischen 1985 und 1998 erhoben. Nach den durch Befragung erhobenen Daten des deutschen Survey 2003 sind 70\% der Männer und 50\% der Frauen übergewichtig und 17\% der Männer sowie 15\% der Frauen adipös (Mensink et al. 2005). Für den Zeitraum 1985-2002 nahm die Häufigkeit der Adipositas bei Männern von 16,2\% auf $22,5 \%$ und bei Frauen von 16,2\% auf 23,3\% zu. Diese Daten entsprechen einer relativen Zunahme von $39 \%$ für Männer und 44\% für Frauen (Prugger u. Keil 2007). Das mittlere Körpergewicht stieg bei Männern um 3,8 kg und bei Frauen um 5,4 kg an (Helmert u. Strube 2004). Neben Alter und Geschlecht variiert die Häufigkeit von Übergewicht stark nach der Region, dem sozialen Status und der Herkunft. Im Osten Deutschlands ist die Prävalenz deutlich höher (Berg et al. 20o1). In Mecklenburg-Vorpommern leben derzeit die „dicksten“ und in Hamburg die „dünnsten“ Deutschen.

Dramatisch ist die Zunahme der Adipositas im Kindes- und Jugendalter. Derzeit wird geschätzt, dass 15\% der Kinder und Jugendlichen im Alter von 3-17 Jahren übergewichtig und 6\% adipös sind (Kurth u. Schaffrath-Rossario 2007). Aus übergewichtigen Kindern werden übergewichtige Erwachsene mit hohem Risiko für adipositasassoziierte Begleiterkrankungen.

\subsection{Bedeutung der Adipositaschirurgie und metabolischen Chirurgie}

Bildete die Chirurgie in der Vergangenheit nur die letzte Option der Behandlung der morbiden Adipositas, ist durch die rasante Entwicklung der minimalinvasiven und endoskopischen Techniken die operative Intervention fester Bestandteil des Therapiekonzepts (Sharma 2004).

\section{Die chirurgische Therapie der Adipositas ist der kon- servativen Behandlung in Bezug auf Effizienz, Aus- maß, langanhaltende Gewichtskontrolle, Vermei- dung von Begleiterkrankungen und Kostenreduktion überlegen.}

Dieser Vorgang wurde auch deswegen beschleunigt, weil bis heute konservative Therapiemaßnahmen im Langzeitverlauf scheitern. Die Pharmakotherapie hat derzeit keine effizienten und sicheren Optionen. Hinzu kommt, dass bei 90\% der Patienten nach einem Gewichtsverlust, der auf konservativem Weg erreicht wurde, eine erneute Gewichtszunahme - häufig über das Ausgangsgewicht - auftritt (sog. Jo-Jo-Effekt).

\subsection{Chirurgische Therapie-Prinzipien}

Mit der Chirurgie werden nur das Symptom Übergewicht und die damit verbundenen Folgeerkrankungen behandelt, jedoch nicht kausal die in ihrer Ätiopathogenese multifaktorielle Krankheit Adipositas. Der Magen ist ein zentrales Organ in der Regulation der Nahrungsaufnahme und immer zentraler Bestandteil der operativen Intervention, die durch gastrointestinale Aus- oder Umschaltungen (Bypässe) ergänzt werden. Die Grundprinzipien sind Restriktion und Malabsorption, die durch hormonelle Regulationen und andere Mechanismen ergänzt und in einem unterschiedlichen Ausmaß kombiniert werden können. Grundsätzlich können folgende Effekte bei den operativen Verfahren unterschieden werden: 1. Restriktion basiert auf dem Grundprinzip, die Zufuhr für feste Nahrung durch eine Verkleinerung des Magenreservoirs einzuschränken. Energiereiche Flüssigkeiten und breiige Kost kann ungehindert aufgenommen werden. Aus diesem Grunde müssen die Patienten eine ausreichende Compliance zeigen, um erfolgreich zu sein.

2. Malabsorption(Malassimilation) wird in erster Linie durch eine späte Vermischung der Verdauungssäfte (Galle- und Pankreassäfte) mit der Nah- 
rung erzielt. Damit wird die Aufnahme von Fett als ein Hauptenergieträger eingeschränkt. Eine kohlenhydratreiche Ernährung schränkt somit den Therapieeffekt ein.

3. Kombinationsverfahren, bei denen beide Prinzipien zur Anwendung kommen, sind zwar von einer exzellenten Gewichtsabnahme gefolgt, doch muss den potenziellen Mangelerscheinungen durch eine intensive Supplementation vorgebeugt werden. Der Magenbypass ist ein vorwiegend restriktives Verfahren, mit milder Malabsorption von Energieträgern und weiteren Effekten. Die biliopankreatische Diversion mit Duodenalswitch (BPD-DS) ist solch ein typisches Kombinationsverfahren (s. Tab. 2).

4. Hormonelle Effekte spielen eine wichtige Rolle bei allen Verfahren, die entweder die Nahrung aus der gastroduodenalen Passage ausschalten (,glucagon-like-peptide“: GLP-1, GLP-6 u. a.) oder das Magenvolumen resezieren (Ghrelin). 80\% der abdominalen Ghrelinrezeptoren befinden sich im Magen, der durch eine Ausschaltung oder Resektion weniger Hungergefühl nach zentral signalisiert.

Alle Effekte können auch kombiniert werden (Weiner 2010).

\section{Restriktion}

Das Prinzip besteht in einer Einschränkung der Nahrungsaufnahme für feste Bestandteile durch eine Verkleinerung des Magenreservoirs. Dazu wird der
Magen in einen Vormagen (Magenpouch) und einen Rest- oder Hauptmagen separiert. Die Nahrungspassage wird durch ein Stoma behindert. Bei rein restriktiven Verfahren, wie dem Magenband, wird das steuerbare Stoma zur Barriere für die Aufnahme von festen Nahrungsbestandteilen (s. Abb. 3).

Die Restriktion wird jedoch nicht bei flüssiger Energieaufnahme wirksam, da Flüssigkeiten das Stoma mühelos passieren können.

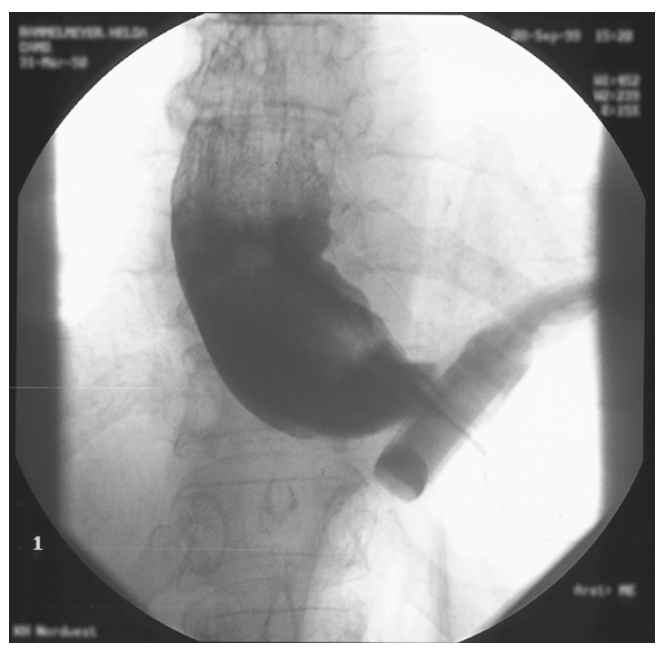

Abb. 3 Röntgendarstellung eines eng eingestellten Magenbandes mit beginnender Dilatation der Speiseröhre („Pelikanphänomen“)

Tab. 2 Chirurgische Prinzipien und typische Verfahren

\begin{tabular}{|c|c|}
\hline restriktive Operation & $\begin{array}{l}\text { steuerbares Magenband („gastric banding“) (s. Abb. 3) } \\
\text { vertikale Gastroplastik mit Bandverstärkung („vertical banded gastroplasty“; VBG) ist kein } \\
\text { aktuelles Verfahren mehr } \\
\text { Schlauchmagen („sleeve gastrectomy“, SG) } \\
\text { proximaler Magenbypass („proximal gastric bypass“, „Roux-en-Y-gastric bypass“; RNYGB) } \\
\text { (s. Abb. 4) hinsichtlich der Nahrungsaufnahme in erster Linie restriktiv }\end{array}$ \\
\hline malabsorptive Operation & $\begin{array}{l}\text { biliopankreatische Diversion („,biliopancreatic diversion“; BPD) } \\
\text { jejunoilealer Bypass (obsolet) }\end{array}$ \\
\hline Kombinationsverfahren & $\begin{array}{l}\text { biliopankreatische Diversion mit Duodenalswitch („duodenal switch“; BPD-DS) } \\
\text { distaler Magenbypass („distal gastric bypass“) }\end{array}$ \\
\hline experimentell & $\begin{array}{l}\text { „Gastric stimulator“ } \\
\text { Duodenalschlauch } \\
\text { Vagusstimulation u. a. }\end{array}$ \\
\hline
\end{tabular}


Beim Magenbypass wird ebenfalls eine Restriktion für feste Nahrungsbestandteile durch eine im Durchmesser limitierte Gastroenterostomie erzeugt. Dazu kommen jedoch vielfältige hormonelle und auch in weitaus geringerem Ausmaß malabsorptive Effekte. Die Restriktion ist jedoch ein Hauptprinzip der Magenbypassoperation, die durch Aufdehnung des Stoma und der alimentären Schlinge wieder verloren gehen kann (Weiner 2010). Das Ausweichen auf hochkalorische („süße“) Getränke wird im Gegensatz zum Magenband durch die potenzielle Entwicklung eines Dumping-Syndroms in den meisten Fällen verhindert. Bei der BPD-DS ist die Restriktion durch den Schlauchmagen ein integraler Bestandteil des Funktionsprinzips, der insbesondere für den initialen Gewichtsverlust verantwortlich zeichnet. Bei der BPD nach Scopinaro wird ebenfalls eine milde Restriktion mit der Bildung eines Magenreservoirs von 200-300 ml erzeugt.

\section{Malabsorption}

Die Nahrungsassimilation ist der Überbegriff für die Digestion (Spaltung höhermolekularer Nahrungsbestandteile in niedrigmolekulare) und Absorption (Aufnahme der niedrigmolekularen Nahrungsbestandteile). Die hohe Kompensations- und Regenerationsfähigkeit des Verdauungstraktes macht eine rasche Adaptation möglich, sodass die Malassimilation nur durch eine massive Einschränkung von Digestion und/oder Resorption möglich wird. Die subtotale Ausschaltung der Resorptionsfläche ist nach den negativen Erfahrungen mit dem Dünndarmbypass in den 197oer-Jahren obsolet. Die Erzeugung der Maldigestion kann durch die späte, d. h. tiefe Einleitung von Verdauungssäften (Gallenflüssigkeit, Pankreassaft) in den nahrungsführenden Darmanteil erzielt werden.

Diese Mechanismen sind unabhängig von der Mitwirkung des Patienten. Allerdings müssen die Mechanismen auch bewusst ausgenutzt werden. Eine alleinige Einschränkung der Nahrungszufuhr verlangt eine Compliance des Patienten, um durch einen „Hungerzustand“ sein Gewicht zu reduzieren. Viele Patienten sind dazu nicht in der Lage, sodass sie unzureichend abnehmen. Durch die Kombination von Nahrungseinschränkung und Fettmangelverdauung wird der Patient durch den eigenen Körper unterstützt. Die Einschränkung in der Fettverdauung geschieht unabhängig vom eigenen Willen und lässt weitaus deutlichere Gewichtsreduktionen erzielen, als eine alleinige Verkleinerung der Aufnahmekapazität. Für extrem übergewichtige Patien- ten (Körpergewicht 225\% über Idealgewicht) und Patienten mit eingeschränkter Compliance sollte die Malabsorption durch biliopankreatische Diversion (BPD) zur Anwendung kommen.

Eine effiziente Malassimilation von Fett als einem Hauptenergieträger wird nur dann erreicht, wenn bei geringer oder nicht existenter Restriktion der ,common channel“ $50 \mathrm{~cm}$ beträgt. Er darf nicht kürzer sein, da sonst profuse chologene Diarrhöen auftreten. In dieser Situation gelangen fast $100 \%$ der Gallensäuren ohne Reabsorption in das Kolon. Langfristig entwickelt sich zu den profusen therapieresistenten Diarrhöen auch ein Gallensäureverlustsyndrom.

\section{Hormonelle Regulationen}

Die komplexen hormonellen Regulationen des Verdauungstraktes, insbesondere der Nahrungsaufnahme sind erst in den letzten Jahren mit der Entdeckung von enterogastralen Hormonen in ersten Ansätzen erforscht worden. Chrelin als das Hungerhormon spielt eine zentrale Rolle in der Steuerung der Nahrungsaufnahme und wird nach den verschiedenen Operationsverfahren unterschiedlich beeinflusst.

Die Ausschaltung duodenaler Rezeptoren, des Hauptmagens insgesamt, aber insbesondere die durch die Dünndarmausschaltung erzeugte hormonelle Diversion mit Veränderung im Sekretionsverhalten einer ganzen Reihe von Enterohormonen ziehen eine Vielzahl metabolischer Veränderungen nach sich, die auch für die Behandlung des metabolischen Syndroms eine Bedeutung besitzen. Ein wichtiger Hinweis für die Bedeutung hormoneller Mechanismen ist die postoperativ sehr kurzzeitige Remission des Diabetes mellitus vom Typ 2 nach allen BypassVerfahren. Die durch die Operation veränderte Regulation von Enterohormonen (GLP-1, GLP-2, „gastric inhibitory polypeptide“ [GIP], Ghrelin u. a.) spielt offenbar eine Schlüsselrolle. Eine adäquate Ausschüttung des GIP verhindert zu hohe Glukosespiegel im Blut. GLP-1 wirkt über eine Stimulation der Freisetzung von Insulin und eine Hemmung der Glukagonsekretion, sodass der Blutzuckerspiegel nüchtern und nach Nahrungsaufnahme verringert ist. Ein Nebeneffekt ist zudem die verzögerte Entleerung von Mageninhalt in den Darm.

Die hormonellen Regulationsmechanismen weisen auch auf den engen Zusammenhang in der Entwicklung von Adipositas und Diabetes mellitus Typ 2. Letztendlich ist die Gewichtsreduktion nicht nur die einzige Prävention, sondern auch die effektive Be- 
handlung des Diabetes mellitus Typ 2. Spezifische Effekte der bariatrischen Operation mit Duodenalexklusion (Bypass-Verfahren, Duodenalswitch), hormoneller Diversion und Fundusresektion (GhrelinEffekt) machen eine „metabolische Chirurgie“möglich, die jeder medikamentösen Therapie oder Insulinapplikation im therapeutischen Effekt überlegen ist. Der Diabetes mellitus vom Typ 2 kann bei Übergewicht und Adipositas durch eine operative Intervention von Bypass-Verfahren schlagartig beseitigt werden, wenn noch rechtzeitig interveniert wird und ausreichend Beta-Zellen vorhanden sind.

\subsection{Indikationsstellung}

Die Indikation $z u$ einem adipositaschirurgischen Eingriff benötigt aufwändige Abklärungen, die durch ein eingespieltes, interdisziplinäres Team von Spezialisten durchgeführt werden sollten. Die Abklärungen beinhalten eine Ernährungsanamnese, das Feststellen der kardiopulmonalen Leistungsfähigkeit und der Ausschluss endokrinologischer Störungen und Stoffwechsel-Erkrankungen. Bereits manifeste Mängel an Vitaminen und Spurenelementen werden präoperativ korrigiert. Die gastroenterologische Untersuchung umfasst immer eine Gastroduodenoskopie zum Ausschluss pathologischer Veränderungen im oberen Gastrointestinaltrakt. Nach einer Bypassoperation können sowohl Restmagen als auch das Duodenum und die Gallenwege endoskopisch nicht mehr untersucht werden. Eine psychiatrische oder psychologische Evaluation zum Ausschluss einer manifesten Psychose oder schweren Essstörungen kann die Abklärungen abschließen, falls anamnestische Anhaltspunkte für eine solche Erkrankung bestehen.

Die Abklärungen für einen adipositaschirurgischen Eingriff erfolgen im interdisziplinären Team unter $\mathrm{Fe}$ derführung des Chirurgen.

1991 wurden von einem Expertengremium Empfehlungen zur Selektion von Patienten für einen bariatrischen Eingriff aufgestellt (National Institutes of Health Consensus Development Conference 1992).

\section{Voraussetzungen zur Durchführung operativer Eingriffe} zur Gewichtsreduktion

- bei erstmaliger Therapie des Übergewichts zunächst Anstreben eines konservativen, nichtchirurgischen Vorgehens
- informierte und motivierte Patienten mit akzeptablem operativen Risiko

- Evaluation durch ein multidisziplinäres Team

- erfahrene Chirurgen in einer Klinik mit adäquater Infrastruktur

- lebenslange medizinische Nachsorge nach der Chirurgie

Daneben gibt es in verschiedenen Ländern Auflagen, die durch die Kostenträger und Leitlinien internistischer Fachgesellschaften erhoben werden. Dabei kommen folgende Kriterien zur Geltung:

- $\mathrm{BMI}>40$

- $\quad$ BMI > 35 mit Begleiterkrankungen, wie arterielle Hypertonie, Diabetes mellitus, SchlafapnoeSyndrom, Dyslipidämie, degenerative Veränderungen des Bewegungsapparates und andere, die durch eine Gewichtsreduktion gebessert werden können.

- Versagen von konservativen Therapieversuchen, die während mindestens 2 Jahren durchgeführt wurden (z.B. ärztlich geführte Diät- und Verhaltensprogramme)

Kriterien wie z.B. willkürlich gezogene BMI-Grenzen, Altersbeschränkungen oder die Voraussetzung einer konservativen und nicht erfolgreichen Therapie sind nicht das Resultat chirurgischer Forschung, sondern von Konsensuskonferenzen und Leitlinien internistischer Adipositasgesellschaften und werden aus diesem Grund heutzutage zunehmend kritisiert.

\section{Fehlende Compliance ist eine Kontraindikation für rein restriktive Verfahren. Die Restriktion gibt nur eine Hilfestellung, die durch den Patienten aktiv um- gesetzt werden muss.}

Ist die Indikation zu einem chirurgischen Vorgehen gestellt, muss das am besten geeignete Verfahren ausgewählt werden. Es gibt kein ideales Verfahren. Alle Techniken haben Vorteile und Nachteile, die abgewogen werden müssen. Diese Auswahl richtet sich nach den Ergebnissen der präoperativen Abklärungen. So hat sich gezeigt, dass Patienten mit einem extrem hohen Gewicht zu wenig von einem rein restriktiven Verfahren profitieren, bleiben doch die meisten dieser Patienten nach einer Bandingoperation auch nach 2 Jahren noch im Bereich der morbiden Adipositas. Weitere Kontraindikationen für ausschließlich restriktive Techniken bilden Essstörungen wie „binge eating disorders“, „sweet eaters"(zwanghaftes Süßessen) und ein insuffizienter unterer Ösophagussphinkter. Auch bei Vorliegen eines 
latenten oder manifesten Diabetes mellitus Typ 2 konnte gezeigt werden, dass ein kombiniertes Verfahren mit Restriktion und Malabsorption der rein restriktiven Methode überlegen ist (Sjöström et al. 2004). Die Steatosis hepatis und Frühformen eines zirrhotischen Umbaus der Leber sind keine Kontraindikationen, sondern unterstützen eher die Indikation für eine operativ induzierte Gewichtsreduktion. Voraussetzung ist eine ausreichende Syntheseleistung der Leber, die sich durch Gewichtsreduktion wieder regenerieren kann.

\subsection{Ergebnisse der Adipositaschirurgie}

\section{Gewichtsverlust}

Im Gegensatz zur konservativen Behandlung können Patienten nach operativen Therapien lang anhaltend ihr Übergewicht um mehr als 50\% reduzieren. Beim Magenband verlieren die Patienten ca. 40-60\% ihres Übergewichts (Buchwald et al. 2004). Dies entspricht einem Abbau von 10-12 BMI-Punkten (Dargent 1999). Nach proximalem Magenbypass verlieren die Patienten etwa $61-77 \%$ des Übergewichts innerhalb von 2 Jahren (Buchwald et al. 2004), beim distalen Magenbypass kann diese Rate auf 9o\% ansteigen (Torres 1991). Nach einer biliopankreatischen Diversion (BPD und BPD-DS) haben die Patienten praktisch kaum Restriktion, verlieren aber dennoch durch die starke Malabsorption 64-74\% ihres Übergewichts (Hess u. Hess 1998); die Folge sind jedoch häufig Diarrhö (14\%) und Steatorrhö mit übelriechendem Flatus.

\section{Auswirkung auf Begleiterkrankungen}

Ebenso wichtig wie der Gewichtsverlust ist die Wirkung auf die Begleiterkrankungen. Das sog. metabolische Syndrom ist ein Hauptfaktor für die Spätmorbidität der Adipositas wie kardiovaskuläre Erkrankungen. Der Diabetes mellitus verbessert sich bei den Bypassverfahren, noch bevor es zu einem signifikanten Gewichtsverlust kommt (Rubino u. Gagner 2002). Komorbiditäten wie die arterielle Hypertonie, Dyslipidämie und das Schlafapnoe-Syndrom reduzieren sich ebenfalls signifikant.

\section{Morbidität und Mortalität}

Die Mortalität für Bypass-Verfahren liegt bei routinierten Teams zwischen o,1 und o,3\% (Schauer et al.
2000) und beim Magenband bei o,05\% (Chapman et al. 2004). Als postoperative Komplikationen treten in erster Linie Wundinfekte auf. Bei Bypassverfahren kommt es in den ersten Jahren selten $\left(1,5^{-2,2} \%\right)$ zur Anastomoseninsuffizienz an der Gastroenterostomie (Schauer u. Ikramuddin 2001), die bei rechtzeitiger Diagnose und raschem Handeln effektiv kontrolliert werden kann. Nach Serien von mehr als 500 bis 1000 Eingriffen pro Operateur tendiert diese Häufigkeit gegen o\%. Daneben kommen postoperative Blutungen einerseits, Lungenembolien trotz Thromboseprophylaxe andererseits vor (Wittgrove u. Clark 2000). Gegenüber der offenen Chirurgie ist die Thrombosehäufigkeit um das 10-Fache niedriger. Die Embolien können noch Wochen nach dem Eingriff auftreten, sodass die Diskussion über die postoperative Dauer der Thromboembolieprophylaxe noch immer nicht abgeschlossen ist.

Im Langzeitverlauf müssen Vitamine $\left(B_{12}, D_{3}\right)$ und Spurenelemente ( $\mathrm{Fe}, \mathrm{Ca}$ ) substituiert werden. Dosierung und Bestandteile richten sich nach Operationsverfahren, Geschlecht und Alter.

Bei rein restriktiven Verfahren sind vermehrt Ösophagusdysmotilitäten und Pouch-Komplikationen (Vergrößerung des proximal des Bandes gelegenen Magenanteils) beschrieben(s. Abb.3) (Gustavsson u. Westling 2002), was zu einem sekundären Bandversagen führen kann. Dies wiederum führt in bis zu 20\% der Fälle nach Magenbanding zu einer Reoperation.

Nach Bypassoperationen mit Verwendung von 21-mm-Zirkulärstaplern werden, häufiger als bei 25-mm-Zirkulärstaplern und Handanastomosen, Anastomosenstenosen gesehen, die sich nahezu immer endoskopisch durch Bougierung beheben lassen.

\subsection{Verfahrenswahl}

Bei der Verfahrenswahl existieren starke geografische Unterschiede. Während in den USA nach der FDA-Zulassung die Magenband-Operationen rasant zunahmen, hat man in Europa die Bandimplantationen weitgehend durch Magenbypassverfahren ersetzt. Die Verfahrenswahl muss jedoch individuell erfolgen. Bei jüngeren Patienten sollten eher reversible Verfahren eingesetzt werden. Es sind dann „Brückenprozeduren“, die entweder in das Erwachsenenalter oder bis zur Verfügbarkeit weniger invasiver Techniken oder pharmakologischer Lösungen für die Betroffenen eine vorübergehende Lösung darstellen. Die 
Implantation von Fremdkörpern sollte im höheren Alter unterbleiben, da hier in den Folgejahren häufiger Revisionseingriffe zu erwarten sind. Die biliopankreatische Diversion ist immer die letzte Lösung.

\section{Laparoskopie versus Laparotomie}

Die Entwicklung der minimal-invasiven Chirurgie (MIC) hat ebenso wie die Zunahme der Adipositas zur Verbreitung der operativen Techniken zur Gewichtsreduktion beigetragen. Besonders profitieren adipöse Patienten von laparoskopischen Operationstechniken, weil aufgrund der Bauchdeckenstärke bei herkömmlichen Operationen ungleich größere Schnitte angelegt werden müssen als bei normalgewichtigen Personen. Außerdem neigen fettreiche Bauchdecken zu Komplikationen wie Blutergüssen, Infektionen und späteren Narbenbrüchen. Bei der minimal-invasiven Chirurgie werden unabhängig vom Körpergewicht die gleichen kleinen Hautinzisionen notwendig. Die Folgen sind geringere Schmerzen und damit frühere Mobilisation, Entlassung und Aufnahme der gewohnten Tätigkeit. Die Komplikationen seitens der Wunden sind weitaus seltener.

Die Laparoskopie hat vor allem bei adipösen Patienten Vorteile gegenüber der offenen Chirurgie (Dindo et al. 2003).

In den USA stieg der Anteil laparoskopischer Techniken von 10\% im Jahre 1999 auf 90\% im Jahre 2004. Dieser Trend hat sich in den letzten Jahren fortgesetzt, ohne die $100 \%$-Grenze zu erreichen. Vergleichende Studien haben für die laparoskopischen Techniken signifikant kürzere Hospitalisationszeiten, weniger postoperative Schmerzen und eine kürzere Rehabilitationszeit nachweisen können (Lujan et al. 2004). Zusätzlich waren die Wundprobleme wie Infektionen $(1,3 \%$ vs. $10,5 \%$ ) und Narbenhernien ( $0 \%$ vs. $7,9 \%$ ) bei der Laparoskopie deutlich geringer. Die höheren Operationskosten für die Laparoskopie wurden durch die niedrigeren Hospitalisationskosten wettgemacht.

\subsection{Operationsverfahren zur Gewichtsreduktion}

\section{Steuerbares Magenband („gastric banding“)}

Das Prinzip des steuerbaren Magenbandes besteht in der alleinigen Restriktion. Durch das Band wird ein kleiner Vormagen (Pouch) gebildet, der durch das einengende Stoma mit dem Hauptmagen verbunden ist. Die innere Oberfläche des Bandes ist aufblasbar. Je nach Füllungszustand ändert sich der innere Durchmesser des Bandes (s. Abb. 3).

Diese Veränderbarkeit des inneren Durchmessers bedingt auch die Steuerbarkeit des Durchlasses (Stoma). Das Band wird mit der Steuerkammer (Port) durch ein langes Silikonband verbunden. Die alleinige Restriktion verlangt eine hohe Compliance des Patienten. Der „excess weight loss“ (EWL) beträgt 45-55\% (Buchwald et al. 2004). Etwa $1 / 3$ der Patienten lässt die notwendige Motivation zur Mitwirkung vermissen. Das Implantat kann mit geringen Operationsrisiken laparoskopisch implantiert werden. Allerdings ist die Re-Operationsrate langfristig höher als $25 \%$, wobei neben einem Verrutschen des Bandes („slippage“) langfristig das Damokles-Schwert der Bandmigration über dem Patienten schwebt. Die Bandkomplikationen treten auch noch Jahre nach der Operation auf.

Das Magenband gilt als ein Standardverfahren, das insbesondere in niedrigeren BMI-Bereichen (< 45) und für junge weibliche Patienten geeignet ist.

\section{Proximaler Magenbypass}

Für den Roux-en-Y-Magenbypass (RNYGB) existieren zahlreiche Modifikationen hinsichtlich der Magenpouchkonstruktion, der gastrojejunalen Anastomose und der Länge der alimentären und biliodigestiven Schlingen. Das Hauptkonstruktionsmerkmal ist ein kleiner Magenpouch, der mit einer alimentären Schlinge anastomosiert ist (s. Abb. 4). Die $50 \mathrm{~cm}$ lange (gemessen vom Treitz-Ligament) biliodigestive Schlinge leitet die Verdauungssäfte $>80 \mathrm{~cm}$ (in den letzten 10 Jahren hat sich die Wahl der Schlingenlänge auf 120-150 cm weltweit durchgesetzt) von der Gastrojejunostomie entfernt in die alimentäre Schlinge, die dann aboral „common channel“ (gemeinsamer Verdauungskanal) genannt wird. Der RNYGB erzeugt im Mittel einen Gewichtsverlust von 60-70\% des Übergewichts (Buchwald et al. 2004).

Hauptmechanismus für den Gewichtsverlust ist Nahrungsrestriktion. Hormonelle Veränderungen in der Regulation (GLP-1-Hormon, Ghrelin) durch Ausschaltung duodenaler Rezeptoren, der Ausschaltung des Fundus (Chrelin) und weitere Prozesse unterstützen nicht nur die Gewichtsreduktion, sondern beeinflussen die Insulinregulation. Damit wird der Diabetes mellitus vom Typ 2 therapeutisch beein- 


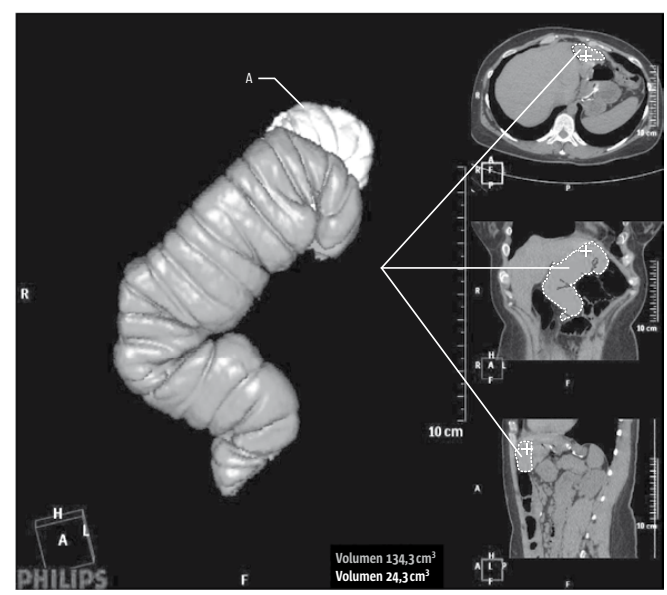

Abb. 4 Virtuelle Pouchografie eines Magenbypass. Kleiner Vormagen (A) ist mit der alimentären Dünndarmschlinge verbunden. Der Restmagen ist nicht sichtbar, da kein Kontrastmedium in den Restmagen eintritt.

flusst und innerhalb von 3 Monaten können $82 \%$ der Patienten ihre Medikation (Insulin u. a.) einstellen (Buchwald et al. 2004). Die malabsorptive Komponente ist für die Entwicklung von Mangelzuständen verantwortlich. Für die Energieaufnahme aus der Nahrung spielt die Malabsorption eine untergeordnete Rolle. Das wird allein dadurch verständlich, wenn man an die Kompensationsfähigkeit des mehrere Meter langen verbleibenden Restdarmes denkt. Eine Resektion von 150 oder $200 \mathrm{~cm}$ Dünndarm verursacht keine dauerhafte Beeinflussung des Körpergewichtes. Die primäre Ringverstärkung des Magenbypass oder die Kombination mit einem steuerbaren Magenband zählt weltweit nicht zum Standard.

\section{Standards sind}

- kleiner Pouch $\left(<15 \mathrm{~cm}^{3}\right)$

- alimentäre Schlinge bis $150 \mathrm{~cm}$

- biliodigestive Schlinge $50 \mathrm{~cm}$ (in Europa)

- Verschluss innerer Hernien zur Vermeidung von Obstruktionen

Die Sicherheit des Verfahrens hängt eindeutig von der Lernkurve(Durchführungshäufigkeit)des Operateurs ab. Die lebenslange Supplementation ist essenziell.

\section{Schlauchmagenbildung („sleeve gastrectomy“)}

Die Resektion eines Großteils des Magens (große Kurvatur) unter Belassung der Magenstraße wird als

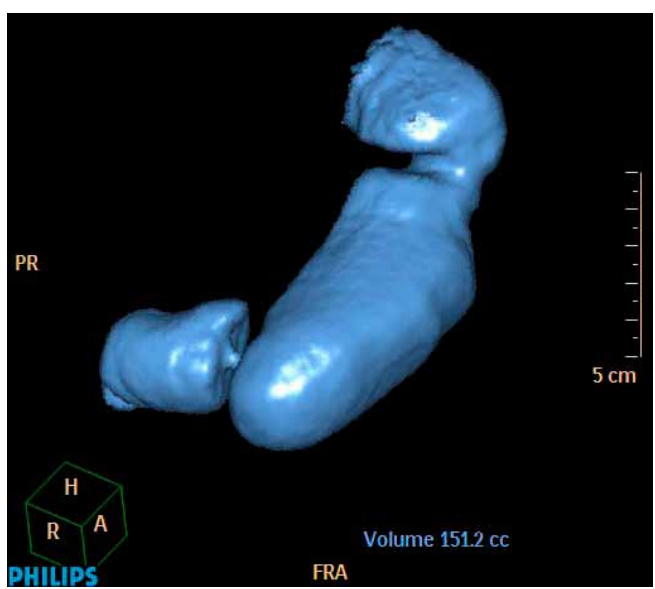

Abb. 5 Virtuelle Darstellung (CT) eines Schlauchmagens

Schlauchmagenbildung oder "Sleeve gastrectomy“ bezeichnet(s. Abb. 5). Diese Operation erfährt in Europa und weltweit eine rasante Verbreitung, da viele $\mathrm{Pa}$ tienten die geringeren Auswirkungen auf den Hormon-und Mineralhaushalt als Vorteil ansehen. Andererseits ist dieses Verfahren im Gegensatz zum Magenbypass irreversibel und ist mit schwerwiegenden Komplikationsmöglichkeiten (Klammernahtleckagen) behaftet.

Die Ausschaltung der Reservoirfunktion des Magens durch die Schlauchmagenbildung hat auch hormonelle Effekte, da die Zahl der Chrelin-Rezeptoren im Magen drastisch verringert wird. Die hormonellen Effekte sind aber nicht dauerhaft, da der Körper auch hier über eine enorme Kompensationsfähigkeit verfügt. Die Gewichtsreduktion erfolgt in erster Linie durch Begrenzung der Nahrungsaufnahme für feste Nahrung und einem reduzierten Hungergefühl. Ein Wiederanstieg des Gewichts nach wenigen Jahren kann dann einen erneuten Eingriff in Form eines Bypass-Verfahrens erforderlich machen.

Die Anpassung der Ernährung an die veränderten Bedingungen stellt das wesentliche Langzeitproblem des Eingriffs dar, der zudem nicht so risikoarm ist, wie man anfangs unterstellt hat. Die mittlere Letalität von $0,4 \%$ weicht gegenüber der Magenbypassoperation mit $0,5 \%$ nur gering von dem komplexeren Eingriff ab, was auf das Fehlen intestinaler Komplikationen hindeutet. Das Verfahren ist noch nicht international standardisiert was Schlauchdurchmesser und Ausdehnung (mit oder ohne Re- 


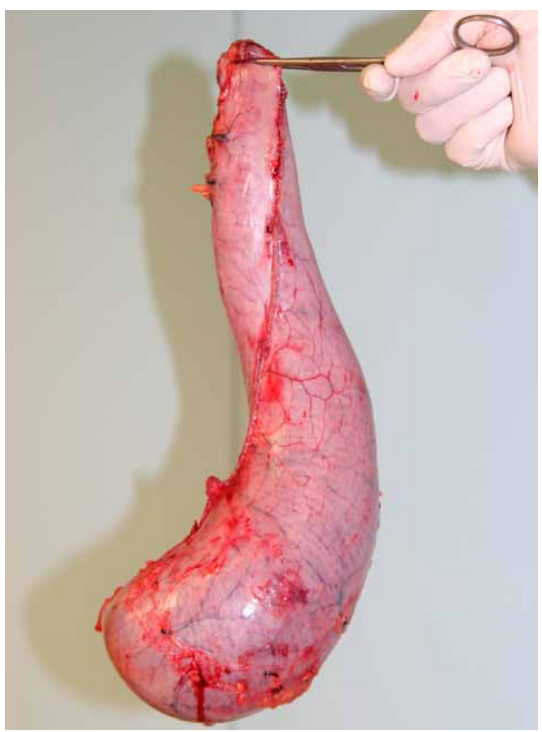

Abb. 6 Magenresektat nach Schlauchmagenbildung

sektion von Antrumanteilen) betrifft. Vergleichende Studien mit Magenbypassoperationen zeigen bezüglich Gewichtsverlust und Veränderungen der Begleiterkrankungen im Kurzzeitverlauf analoge Ergebnisse. Allerdings ist der gastroösophageale Reflux nach LSG deutlich häufiger.

Die Schlauchmagenbildung ist ein Verfahren, das rasch eine Verbreitung findet, weil es sich bei guten kurz- und mittelfristigen Ergebnissen hinsichtlich der Gewichtsreduktion operationstechnisch „simpel“ darstellt. Es ist im Gegensatz zu Magenband und Magenbypass irreversibel (s. Abb. 6), weshalb die Aufklärung und Entscheidungsfindung diese Aspekte berücksichtigen muss.

Es handelt sich um ein etabliertes Verfahren zur Mehrschritttherapie mit dem ungeklärten Problem der Klammernahtleckagen im His-Winkel und dadurch bedingter Morbidität und Mortalität, die knapp unter denen der Magenbypassoperationen liegen. Eine Supplementation wird angeraten, da bereits Mangelerscheinungen berichtet wurden. Etwa $1 / 3-2 / 3$ der Patienten benötigen nach 5-10 Jahren einen Zweiteingriff (Weiner et al. 2007).

\section{Biliopankreatische Diversion (BPD)}

Die biliopankreatische Diversion (BPD) mit Ausschaltung des Dünndarms von einer gemeinsamen Passa- ge von Nahrung und Verdauungssäften mit einer gleichzeitigen Magenverkleinerung wurde von Scopinaro (Scopinaro et al. 1979) entwickelt. Das Restvolumen des Magenpouches muss jedoch 200$300 \mathrm{~cm}^{3}$ betragen, was eine geringe Restriktion darstellt. Ein verbleibender gemeinsamer Verdauungskanal von nur $50 \mathrm{~cm}$, in dem sich Verdauungssäfte (Gallen- und Pankreassaft) mit der Nahrung mischen (auch als „common channel“ bezeichnet), führt zu einer Malassimilation von Fett. Die biliodigestive Schlinge ist lang, da bei der Abmessung der alimentären Schlinge, beginnend von der Bauhin-Klappe, die Darmkontinuität nach insgesamt $250 \mathrm{~cm}$ durchtrennt wird.

Die Modifikationen betreffen in erster Linie die Länge der biliodigestiven und damit auch der alimentären Schlinge. In der Modifikation nach Larrad et al. (2007) wird die biliodigestive Schlinge ( $\mathrm{BL}=$ biliodigestive limb) mit $50 \mathrm{~cm}$ kurz gehalten, der „common channel“ bleibt standardmäßig bei $50 \mathrm{~cm}$. Der Eingriff wird daher auch als Larrad 50-50 BPD bezeichnet.

In der Originaltechnik BPD-Scopinaro wird der Restmagen entfernt. In der Ära der MIC wird in einigen Zentren auf die Restgastrektomie verzichtet. In diesen Fällen muss eine mindestens 18-monatige medikamentöse Magensäurehemmung durchgeführt werden, um die Bildung von Ulzera im Restmagen und im Duodenum zu verhindern. BPD ist die letzte Option nach Versagen oder Rückfall nach restriktiven Verfahren.

\section{Die BPD ist die einzige langfristig in der Gewichtskur- ve stabile und effiziente Operation und benötigt um- fassende Supplementation.}

\section{Biliopankreatische Diversion mit Duodenalswitch (BPD-DS)}

Diese Technik ist in ihrer Gewichtsreduktion dem BPD gleichwertig, allerdings mit dem Vorteil des Erhalts des Pylorus. Es erfolgt eine Schlauchmagenbildung, die jedoch nicht so restriktiv sein darf wie ein Magenbypass (Sleeve-Durchmesser: Charr 54-6o). Das Duodenum wird postpylorisch (unter Erhalt des Pylorus) durchtrennt und mit der alimentären Ileum-Schlinge anastomosiert. Die biliodigestive Schlinge leitet die Verdauungssäfte $75-100 \mathrm{~cm}$ von der Bauhin-Klappe entfernt in das Ileum. Ein DumpingSyndrom tritt durch Erhalt des Pylorus nicht auf. Die gastrointestinalen Nebenwirkungen in den ersten 
Monaten nach der Operation sind zunächst schwerwiegender als nach einem RNYGB, da der Schlauchmagen anfangs nur verzögert transportiert und der Darm sich funktionell adaptiert. Es gibt eine Reihe von technischen Variationen (Weiner et al. 2004).

Mit der Verbreitung der Schlauchmagenbildung tritt der BPD-DS in einer Prozedur zurück und wird vorwiegend als zweiter Schritt nach LSG eingesetzt. Die Letalität ist höher als bei allen anderen Verfahren. Sie beträgt in der Metaanalyse von Buchwald et al. (2004) 1,1\%. Bei einem BMI von über 6o steigt sie auf $6 \%$ an. Mit dem Erhalt des Pylorus werden die Gefahren eines späteren Dumping-Syndroms umgangen. Allerdings beinhaltet die Durchtrennung des Zwölffingerdarms die gefürchtete Komplikation der Duodenalstumpfinsuffizienz. BPD-DS ist die komplexeste Operation mit der höchsten Morbidität und Mortalität, allerdings dafür mit der besten Lebensqualität. Primäre BPD-DS-Operationen sind selten geworden, bei einem BMI > 55 sollten sie stets als Zweischrittoperation durchgeführt werden (Weiner 2009).

\section{Omega-Loop-Magenbypass}

Der Magenpouch wird durch Längsresektion des Magens entlang einer Kalibrationstube deutlich größer als beim „klassischen Bypass“. Eine oral oder nasal platzierte Magensonde stellt eine Leitschiene für eine Resektion dar. Durch die Wahl des Durchmessers wird der spätere Pouch kalibriert (daher der Begriff „Kalibrationstube“). Es ist faktisch ein kurzer Schlauchmagen, an dem großkurvaturseitig eine ungeteilte Dünndarmschlinge angeschlossen wird. Die Dünndarmschlinge wird als Seit-zu-seit-Anastomose ohne Fußpunkt- oder Braun-Anastomose angeschlossen, weshalb ein Kontakt der Anastomose mit der Galleflüssigkeit unausweichlich ist. Die biliodigestive Schlinge ist $200 \mathrm{~cm}$ lang. Die Anastomose wird am kaudalen Ende des schlauchförmigen Magenpouches in Seit-zu-Seit-Technik angelegt. Bei Patienten, die eine Bluttransfusion ablehnen, ermöglicht der BII-Magenbypass bei postoperativen Anastomosenblutungen die endoskopische Blutstillung im Bereich der Gastroenterostomie. Das Risikopotenzial der Blutung aus der Entero-Entero-Anastomose entfällt.

Bei extremer Lebervergrößerung kann die hiatale Region nicht unter Sicht erreicht werden. Hier kann eine schlauchfömige (analog Schlauchmagenbildung, „sleeve gastrectomy“) Pouchbildung mit einer Resektion entlang einer Kalibrationssonde einen weit nach kaudal reichenden Pouch bilden, bei dem die Gastroenterostomie unter Sicht weit kaudal ausgeführt wird. Bei Hochrisikopatienten kann die Operationszeit verkürzt werden. Der Omega-LoopMagenbypass ist eine Stagingoperation, die bei Hochrisikopatienten und bei extremem BMI durchgeführt wird. Die Umwandlung in eine Roux-Y-Form kann später nach Gewichtsverlust wegen Gallereflux einfach und mit erniedrigtem Risiko erfolgen.

\section{Distaler Magenbypass}

Die Kombination von Magenpouch und kurzem Darmschenkel für die Verdauung (Common channel, CC) initiiert eine überwiegend malabsorptive Komponente. Die verbleibende Darmlänge des CC wird von der Bauhin-Klappe auf 50-100 cm gemessen. Eine Reihe von Chirurgen wählen auch einen CC von mehr als $100 \mathrm{~cm}$. Langfristig können sich neben den bekannten Mangelerscheinungen des proximalen Magenbypass zusätzlich Proteinmangelzustände entwickeln, da aufgrund der unzureichenden Verdauung von Proteinfasern die Absorption von Eiweißbausteinen im kurzen Restdarm eingeschränkt wird. Proteinmangelzustände sind faktisch kaum zu behandeln und machen regelmäßige intravenöse Supplementationen notwendig. Der distale Magenbypass zählt daher in Europa nicht zu den Standardverfahren, die allgemein empfohlen und eingesetzt werden. In den USA ist der distale Magenbypass mit weniger als $8 \%$ der Bypass-Verfahren ebenfalls nicht sehr stark verbreitet (Buchwald et al. 2004).

\section{Magenschrittmacher}

Es existiert derzeit kein effektives Verfahren der Magenstimulation zur Gewichtsreduktion. Der Einsatz beim Diabetes mellitus kann bei einigen Patienten eine Senkung des HbAlc-Wertes um 1\% bewirken.

\section{Fettabsaugung (Liposuktion)}

Die Fettabsaugung ist keine Adipositaschirurgie, sondern ein Verfahren der plastischen Chirurgie, das zur lokalen Entfernung überschüssiger Fettdepots eingesetzt werden kann. Es ist für die Behandlung der Adipositas grundsätzlich nicht geeignet. Ein Nutzen dieser Technik für die langfristige Gewichtssenkung ist nicht belegt, die Risiken dieses Eingriffs sind schlecht dokumentiert, aber nicht unerheblich. 


\subsection{Vergleich der Operationsverfahren hinsichtlich des Gewichtsverlustes}

Die Ergebnisse von Metaanalysen (Buchwald et al. 2004) und vergleichenden Studien aus dem Jahr 2009 (Strain et al. 2009) gleichen sich, wenn es um den Gewichtsverlust nach verschiedenen Operationsverfahren geht.

In allen Publikationen kommt es übereinstimmend zu einer folgenden Rangfolge des Gewichtsverlustes: BPD-DS (Biliopankreatische Diversion) $\rightarrow$ RNYGB (Magenbypass) $\rightarrow$ LSG (Schlauchmagen) $\rightarrow$ LAGB (Magenband)

\subsection{Komplikationen und Management}

Morbid Adipöse sind Hochrisikopatienten, die meist neben Folgeerkrankungen über eine eingeschränkte kardiopulmonale Kapazität verfügen. Die Eingriffe selbst sind Hochrisikoeingriffe, da sie in der hiatalen Region mit Resektionen und Anastomosenbildung ein großes Gefahrenpotenzial in sich bergen. Ein ausreichendes Training des Chirurgen vor dem Start mit der Adipositaschirurgie ist absolute Notwendigkeit. Die Durchführung adipositaschirurgischer Eingriffe verlangt eine außergewöhnlich enge Zusammenarbeit mit dem Anästhesisten. Diese beginnt bei der Prämedikation und Indikationsstellung, setzt sich bei der Patientenlagerung und auch Umlagerung während einer Operation (BPD-DS) fort und schließt die Verwendung von Kalibrationssonden, Dichtigkeitsprüfungen (Blauprüfungen, Gasinsufflation) und intraoperativen Gastroskopien ein. Besonders Sondenkomplikationen (s.u.) sind potenzielle Gefahren bei der laparoskopischen Magenchirurgie und müssen unbedingt vermieden werden.

Konversion: Der Verfahrenswechsel, d. h. die Konversion von einer videoendoskopischen minimal-invasiven Vorgehensweise in eine Laparotomie (Bauchschnitt) ist integraler Bestandteil der Aufklärung. Intraoperativ muss aus folgenden Cründen rechtzeitig ein Verfahrenswechsel eingeleitet werden, wenn Komplikationen auftreten, die minimal-invasiv nicht zu beherrschen sind:

- technische Unzulänglichkeit oder extreme intraabdominelle Fettansammlung lassen eine sichere Ausführung der Operation unter videoendoskopischen Bedingungen nicht zu

- Zeitdauer des Eingriffs überschreitet präoperativ gesetzte Limits
Wichtig ist, dass diese Entscheidung rechtzeitig getroffen wird, um Schaden vom Patienten fernzuhalten. Wird diese rasche Entscheidung nicht getroffen und es entwickeln sich durch zögerliches Abwarten schwere Komplikationen, die mit Folgeschäden oder Tod des Patienten einhergehen, so wird diese Fehlentscheidung dem Operateur negativ ausgelegt werden. Die operativ-technischen Voraussetzungen für eine Fortführung der Operation per laparotomiam müssen immer gewährleistet sein (Assistenz, extralange Haken und Instrumente).

\section{Sondenkomplikationen}

Am häufigsten kommt es zum Erfassen von Sonden mit dem Linearstapler, wenn die Sonde nicht rechtzeitig oder vollständig bei Resektionen zurückgezogen wurde. Weiterhin werden mehrfache Sondierungen notwendig, wenn Sondengrößen gewechselt werden. Hier kann es auch zu Perforationen des Ösophagus, der Pouchhinterwand oder auch Anastomose kommen. Im Gegensatz zu offenen Operationen kann keine manuelle Führung der Sonden erfolgen und das Geschehen kann lediglich auf dem Videomonitor beobachtet werden.

\section{Blutungskomplikationen}

Blutungskomplikationen sind bei komplexen Operationen, insbesondere bei Verwendung von Klammernahtgeräten sehr vielfältig. Die Entscheidung, ob konservatives Vorgehen mit Abwarten, Transfusionen, Relaparoskopie oder Relaparotomie gewählt werden, hängt von der Intensität, Kreislaufreaktion, klinischen Symptomatik und der Lokalisation der Blutung ab. Bei der Auswahl von Operationsverfahren sollten bei Patienten, die die Transfusion von Blut und Blutderivaten ablehnen, möglichst nichtresezierende Techniken ausgewählt werden.

\section{Insuffizienzen}

Die Diagnostik von Komplikationen bei morbid Adipösen ist sehr schwierig. Tachykardie, Fieber und Atemnot sind oftmals die ersten und einzigen Hinweiszeichen auf Leckagen nach Bypassoperationen, weshalb nur die umgehende Relaparoskopie bei allen Auffälligkeiten die Entwicklung von lebensbedrohlichen Zuständen verhindern kann. Röntgenkontrastuntersuchungen und CT sind bei extremer 
Adipositas oftmals nicht möglich oder in ihrer Aussagekraft eingeschränkt. Revisionseingriffe wegen Anastomoseninsuffizienz können „offen“ oder laparoskopisch durchgeführt werden. Klammernahtblutungen können konservativ behandelt werden.

\section{Lungenembolien}

Die Lungenembolie (LE) ist eine allgemeine und möglicherweise tödliche Komplikation. Adipositas ist ein unabhängiger Risikofaktor für LE-Ereignisse. Adipositaschirurgie-Patienten haben ein hohes Risiko für die Entwicklung einer LE. Die Häufigkeit einer perioperativen Lungenembolie (LE) ist auf o, 85-1,2\% geschätzt worden (de Freitas et al. 2006).

Thrombo-embolische Ereignisse bleiben die führende Todesursache in der Adipositaschirurgie, wenn sie auch in der Ära der minimal-invasiven Chirurgie und Thromboseprophylaxe sehr selten geworden sind.

\section{Rhabdomyolyse}

Die Rhabdomyolyse ist eine weitere gefürchtete Komplikation, die besonders bei extrem adipösen Patienten auftreten kann. In der Adipositaschirurgie wird die Rhabdomyolyse als Folge des hohen Druckes auf die Muskulatur auf dem Operationstisch mit Ausbildung eines Kompartmentsyndroms angesehen.

Generell sind allgemeine Komplikationen und damit die Operationsmorbidität in den letzten Jahren stets weiter gesenkt worden. Insbesondere die Minimierung der Zugangswege hat zu einer Senkung der allgemeinen Komplikationen beigetragen.

Auf die spezifischen Komplikationen und die Auswirkungen der operativ induzierten Cewichtsreduktion auf die Komorbiditäten und Folgeerkrankungen kann hier nicht detailliert eingegangen werden. Crundsätzlich kann jedoch festgestellt werden, dass die positiven Effekte den Nebeneffekten wesentlich überlegen sind.

\subsection{Nutzung natürlicher Körperöffnungen („Natural orifice transluminal endoscopic surgery, N.O.T.E.S.)}

Die Nutzung natürlicher Körperöffnung als Zugang zu den Organen der Bauchhöhle mit Minimierung des Traumas der Bauchdeckenpenetrationen erscheint auch für die Adipositaschirurgie attraktiv. Gegenwärtig erscheinen die Schlauchmagenbildung und der alleinige Bypass ohne Resektion (Gastroenterostomie) als durchführbare Eingriffe. Generell ist derzeit zu konstatieren:

- N.O.T.E.S. wird die technologische Entwicklung stimulieren und zur Entwicklung neuartiger Geräte und Operationsroboter führen.

- Der transgastrale Zugang hat gegenüber dem transvaginalen Zugang die größeren Aussichten für die Entwicklung viszeralchirurgischer Eingriffe durch N.O.T.E.S.

- Transvaginale oder endoskopische (gastroskopische) Schlauchmagenbildungen haben bislang keine wesentlichen Vorteile gegenüber einer laparoskopischen oder „single-port technique“ nachweisen können.

\section{Neue Zugangswege ab 2009:}

\section{"Single incision laparoscopic surgery"; S.I.L.S.}

Die Minimierung der Schnittführung durch nur einen Zugang (,single incision laparoscopic surgery“; S.I.L.S.) kann die Kosmetik, jedoch nicht immer das postoperative Schmerzempfinden verbessern. Die Operationszeiten verlängern sich in der Anfangsphase deutlich und bleiben auch nach der Lernphase hinter denen der Mehrfachtrokar-Technik zurück. Der kosmetische Aspekt spielt in der Adipositaschirurgie eine geringere Rolle als in der allgemeinen Viszeralchirurgie normalgewichtiger Patienten, zumal der zu bevorzugende infraumbilikale Zugang nicht immer für die Operationsdurchführung im hiatalen Bereich geeignet ist. Es sind alle gängigen Operationsverfahren, mit Ausnahme der malabsorptiven, bereits in S.I.L.S.-Technik durchgeführt worden. Inwieweit sich diese Zugangstechnik in der Adipositaschirurgie verbreiten wird, bleibt abzuwarten. In dieser Sparte der Hochrisikochirurgie stehen die Sicherheit der Operationsdurchführung und kurze Operationszeiten zur Vermeidung allgemeiner Komplikationen (Lungenembolie, Rhabdomyolyse, Dekubitalulzera u.v.a.m.) im Vordergrund. Bei der Schlauchmagenbildung ergibt sich der große Vorteil, dass das Magenresektat über die erweiterte Einzelinzision dann auch ohne zusätzliche Schnitterweiterung entfernt werden kann. Ein Vorteil, der auf die N.O.T.E.S.-Technik nicht zutrifft. 


\subsection{Metabolische Chirurgie}

Die gravierenden Veränderungen von präoperativ vorhandenen Stoffwechselstörungen nach adipositaschirurgischen Eingriffen haben dazu geführt, dass der Begriff „,metabolische Chirurgie“ eingeführt wurde. Folgerichtig haben sich die internationalen Fachgesellschaften, angefangen von der American Society for Metabolic and Bariatric Surgery (ASMBS) bis hin zur internationalen Föderation für Adipositaschirurgie dementsprechend umbenannt. Von metabolischer Chirurgie wird gesprochen, wenn die Behandlung des Diabetes mellitus Typ 2 (DMT2) oder anderer schwerwiegender Stoffwechselstörungen im Fokus der Indikation zur operativen Intervention steht. Damit rücken das Übergewicht und der BMI als alleiniger Maßstab für die Indikationsstellung zur Operation in den Hintergrund, denn die Effizienz der Maßnahmen ist auch bei geringem Übergewicht und niedrigen BMI-Klassen gegeben.

Die Leitlinien zur Adipositaschirurgie setzen die Grenze für die Indikation zum operativen Eingriff beim Vorliegen von Folgeerkrankungen des Übergewichtes, die durch eine Gewichtsreduktion wesentlich gebessert werden können, auf den Grenzwert von 35 fest. Dabei wurde in der neuen S3-Leitlinie (DGAV 2010) festgestellt, dass diese Behandlung auch bei einem niedrigen BMI hinsichtlich der Stoffwechselfunktion, aber auch der Gewichtsreduktion effektiv ist. Sie sollte jedoch nur im Rahmen von Studien durchgeführt werden. Auf der Basis der Erkenntnisse der letzten Jahre werden in verschiedenen Ländern nunmehr auch die Indikationserweiterungen im Rahmen von Studien vorgenommen. Hier ist jedoch mit weiterer fundierter Datenlage eine grundlegende Veränderung zu erwarten. Besonders in Indien, wo durch die genetische Disposition der DMT2 wesentlich früher, d.h. bei einem niedrigen BMI auftritt, werden die Indikationen bereits zum jetzigen Zeitpunkt weit unter der Grenze eines BMI von 35 gesetzt.

\subsection{Mehrschritttherapie}

Adipositas ist mit einer Vielzahl von Folge- und Begleiterkrankungen assoziiert, die diese Patienten zu Hochrisikofällen für jeden chirurgischen Eingriff werden lassen. Die Einschränkungen der kardiopulmonalen Leistungsfähigkeit sind teilweise derartig gravierend, dass eine Vielzahl der Patienten auch schon in jungen Lebensaltern in die ASA-Klassifikation $\geq$ III eingestuft werden müssen. Mit einer guten präoperativen Konditionierung, einer angepassten
Narkoseführung, kurzen Operationszeiten und rascher Mobilisation lässt sich die Mehrzahl der Patienten auch mit hohen BMI-Klassen operieren. Das Risiko besteht im Auftreten von Komplikationen, die diese Patienten mit ihren drastisch eingeschränkten oder fehlenden Reservekapazitäten vital besonders gefährden können. Bereits geringfügige Komplikationen, die ein normalgewichtiger gesunder Patient weitgehend für kurze Zeiträume kompensieren kann, führen bei diesen Patienten zur Dekompensation und vitalen Gefährdung.

Die präoperative Verbesserung der Ausgangssituation ist daher eine wichtige Maßnahme zur Risikominimierung. Die präoperative Gewichtsreduktion nimmt dabei eine Schlüsselrolle ein und verbessert zudem die Bedingungen für eine operative Intervention auch in technischer Hinsicht (verkleinertes Lebervolumen, Verluste an intraabdominellem Fett) wesentlich. Potenzielle Nebeneffekte, wie eine präoperativ induzierte Katabolie mit ihren negativen Auswirkungen auf die Immunabwehr, der Produktion von kurzlebigen Funktionsproteinen für die Wundheilung und anderen Organfunktionen müssen mitberücksichtigt werden. Die katabole Situation kann nicht umgangen, die Intensität der Proteolyse kann jedoch protektiv durch gezielte Ernährung und körperliche Aktivität eingeschränkt werden. Dennoch ist bei Patienten mit einem BMI $>$ Go oder einem absoluten Körpergewicht von mehr als $200 \mathrm{~kg}$ die Überlegung berechtigt, ob der adipositaschirurgische Eingriff nicht in mehreren Schritten durchgeführt oder mit einer präoperativen $\mathrm{Ma}$ genballontherapie begonnen werden sollte.

\subsection{Nachsorge}

\section{Rationale für eine lebenslange Nachsorge}

Wesentliche Voraussetzung für eine erfolgreiche Therapie der Adipositas und ihrer Begleiterkrankungen ist die lebenslange Nachsorge der Patienten. Hierin liegt eine spezifische Besonderheit der Adipositaschirurgie, die sich grundlegend von allen anderen elektiven chirurgischen Operationsverfahren mit Ausnahme der Organtransplantation unterscheidet. Das Nachsorge-Management ist an ein Netzwerk gebunden, welches dem Patienten eine ortsnahe Betreuung ermöglicht, die jedoch von dem Adipositaschirurgen zentral geführt und überwacht werden sollte. Die Kontrolle des Gewichtsverlaufes, die Veränderung der Komorbiditäten und die Früherkennung von Langzeitkomplikationen werden in den Nachsorge- 
sprechstunden erfasst und dokumentiert, evaluiert und behandelt. Darüber hinaus erfolgt die Datensicherung und Eingabe in die zentrale Qualitätssicherung für Adipositaschirurgie in Deutschland.

Da die krankhafte Adipositas nicht kausal therapiert wird, und es sich um eine chronische Erkrankung handelt, muss die Nachbetreuung lebenslang erfolgen. Einen kurativen Behandlungsansatz für die Adipositas gibt es derzeit nicht. Der Schwerpunkt bleibt eine gesamtgesellschaftliche Aufgabe in der Prävention. Es ist unbestritten, dass die Nachsorge nur von fachkompetenten Ernährungsmedizinern und Chirurgen durchgeführt werden kann, die eine detaillierte Kenntnis der einzelnen eingesetzten Operationsverfahren und -materialien besitzen. Ohne lebenslange Nachsorge entwickeln viele Patienten Mangelerscheinungen, Langzeitkomplikationen oder haben durch eine erneute Gewichtszunahme aufgrund einer Ernährungsumstellung oder einer chirurgischen Komplikation den therapeutischen Effekt verloren. Das lebenslange Follow-up für die operierten Patienten ist eine conditio sine qua non für die Adipositaschirurgie.

\section{Gegenstand der Nachsorge}

Bei der Nachsorge soll der Gesamtgesundheitszustand des Patienten beurteilt werden. Der Therapieverlauf wird nicht nur durch die Erfassung des aktuellen Körpergewichts auf einer geeichten elektronischen Waage, sondern auch durch die aktuelle Erfassung des Übergewichtsverlustes und des BMI dokumentiert. Dazu ist die Bewegung des Patienten unter Kontrolle vorzunehmen und sofort zu dokumentieren. Die wiederholte Messung der Körpergröße ist ebenfalls im Langzeitverlauf notwendig, da sich hier ebenfalls Veränderungen ergeben. Das trifft insbesondere auf Kinder und Jugendliche zu, jedoch auch auf Patienten jenseits des 50. Lebensjahres. Die vitalen Körperfunktionen einschließlich des Essverhaltens, der Stuhlgangfrequenz und -beschaffenheit zählen ebenso zur Anamnese wie die allgemeinen Lebensbedingungen in psychosozialer Hinsicht.

\section{Veränderung der Komorbiditäten und der Folgeerkrankungen}

Sie sind sorgfältig zu untersuchen und zu dokumentieren, insbesondere hinsichtlich der veränderten Medikation. Bei notwendigen Veränderungen hinsichtlich der Einstellung des Diabetes mellitus, der
Bluthochdruckkrankheit oder anderer Folgeerkrankungen muss der Hausarzt kontaktiert werden. Die Verwendung der CPAP-Masken muss fortlaufend überprüft werden, ehe diese Behandlung in vielen Fällen eingestellt werden kann.

\section{Vermeidung von Mangel- und Fehlernährungen}

Die Ernährungsanamnese ist ein wichtiger Bestandteil der Nachsorge, die frühzeitig die Entstehung von Fehlentwicklungen aufdecken soll. Bei Verdacht auf Veränderung der Ernährungsweise hinsichtlich einer einseitigen Ernährung (z.B. in flüssiger Form, restriktive Verfahren) muss diese in Einzelfällen auch durch Ernährungsprotokolle subtil erfasst werden. Die Beurteilung des Zahnstatus ist sowohl vor als auch nach Operation ein wichtiger Bestandteil, um eine vollwertige und ausreichende Ernährung zu gewährleisten. Patienten mit restriktiver Form müssen ausreichend gut kauen können, um feste Nahrung zu sich zu nehmen und ein Ausweichen auf pürierte Kost oder Flüssignahrung auszuschließen. Die Entwicklung eines Sweet-eater-Syndroms beim Magenband zeigt sich oftmals in einem kariösen Zahnstatus.

\section{Früherkennung von chirurgischen Komplikationen}

Die Symptomatik von chirurgischen Komplikationen kann sehr vielfältig sein und ist von den Operationsverfahren abhängig. Nach puren restriktiven Verfahren ist ein Gewichtsanstieg entweder durch Änderung der Ernährungsform oder Aufhebung der Restriktion verursacht. Beim Magenband kann diese Gewichtszunahme das Zeichen einer Bandmigration oder auch eines Funktionsverlustes des Magenbandes sein. Röntgendiagnostik und Endoskopie liefern die Diagnose und stellen die Weichen für eine operative Intervention.

Intermittierende Bauchschmerzen, die uncharakteristisch sind und bis in den linken Oberbauch aber auch in den Rücken ausstrahlen, Unregelmäßigkeiten in der Stuhlfrequenz oder kolikartige Unterbauchbeschwerden können durch innere Herniationen oder Adhäsionen der Dünndarmschlingen nach Bypassverfahren auftreten. Bei intermittierend auftretenden Schmerzen ist daher die erneute Laparoskopie und Inspektion aller potenziellen inneren Hernien die einzige Möglichkeit, um eine potenzielle Inkarzeration des Dünndarms mit nachfolgender Nekrose und Ischämie zu vermeiden. 


\section{Erfassung von Mangelzuständen}

Klinisch manifeste Mangelzustände von Mineralstoffen, Spurenelementen und Vitaminen entwickeln sich erst über einen längeren Zeitraum, sodass sich eine allgemeine Labordiagnostik in den ersten Monaten nach adipositaschirurgischen Eingriffen auf die Kontrolle des Hämoglobins und der Entzündungsparameter und ggf. Leberwerte beschränken kann. Die Anämie, die insbesondere bei menstruierenden Frauen nach Operationen mit Ausschluss des Duodenums im Rahmen von Bypass-Verfahren auftreten kann, äußert sich in den allgemein bekannten Symptomen. In der laborchemischen Diagnostik wird dann nicht nur das Hämoglobin sondern auch gleichzeitig der Eisenspiegel und der Vitamin- $\mathrm{B}_{12}{ }^{-}$ Spiegel ermittelt. Die Erfassung von Osteoporosen durch einen sekundären Hyperparathyreoidismus ist insbesondere bei weiblichen Patienten jenseits der Menopause häufig und kann durch eine Parathormonbestimmung und Knochendichtemessung objektiviert werden. Die Therapie mittels Osteoporoseschema mit Vitamin $\mathrm{D}_{3}$ und Kalzium muss langfristig und dauerhaft durchgeführt werden und ist von der Normalisierung der Parathormonwerte unabhängig.

Vielfältiger sind die Symptome von neurologischen Erkrankungen, die durch Mangel an B-Vitaminen auftreten. Diese sind nach allen Operationsverfahren mit und ohne Malabsorption möglich. Der Verlust von Kopfhaaren ist ein normales Symptom nach massiver Gewichtsreduktion, wie es auch im Rahmen von Diätprogrammen zu verzeichnen ist. Viele Patienten klagen in den ersten 3-6 Monaten nach der Operation über vermehrten Haarausfall. Dies ist allerdings ein temporärer Prozess. Die Bildung von Gallenkonkrementen ist ebenfalls ein häufiges Folgesymptom nach Gewichtsreduktion, das der sonografischen Kontrolle bedarf. Die prophylaktische Gabe von Medikamenten wird in der Effizienz unterschiedlich beurteilt. Sie sollte nur in der Phase der starken Gewichtsreduktion durchgeführt werden.

\section{Lebensqualität}

Die Erfassung der Lebensqualität durch spezielle Test- und Fragebatterien ist integraler Bestandteil der Erfassung der postoperativen Veränderungen und dokumentiert in der Regel eine allgemeine grundlegende Verbesserung der Lebenssituation des Patienten (Weiner et al. 2008).

\section{Psychosozialer Support}

Viele Patienten waren vor der Operation bereits über Jahre oder Jahrzehnte hinweg psychologisch stigmatisiert. Die sich daraus entwickelnden psychologischen Störungen bis hin zur hartnäckigen Körperschemastörung bedürfen einer langfristigen Therapie und Unterstützung, insbesondere nach der Operation, wenn sich das Körperäußere ändert. Fortbestehende Erkrankungen, wie Bulimie, können postoperativ erneut auftreten.

\subsection{Folgeoperationen}

Plastisch-ästhetische Eingriffe zählen zu den häufigsten Folgeoperationen. Sie werden insbesondere dann notwendig, wenn eine starke Gewichtsreduktion erfolgt. Besonders tritt ein Hautüberschuss ein, wenn erst im höheren Alter (geringere Hautelastizität) und bei hohem BMI operiert wird. Junge Menschen, die sich bei einem BMI $\leq 40$ einem adipositaschirurgischen Eingriff unterziehen, benötigen selten einen derartigen Folgeeingriff aus medizinischer Indikation. Die Operationszeiten und der Blutverlust sind bei diesen Eingriffen um ein Vielfaches höher, als bei dem adipositaschirurgischen Eingriff.

\subsection{Revisionseingriffe}

Der Verlust der Restriktion ist die häufigste Indikation für einen Jahre später erforderlichen Korrektureingriff. Die Einlage eines Ringes oder die Nachresektion dilatierter Anteile von Magen und Dünndarm sind einfache Eingriffe, die bei entsprechender Erfahrung des Operateurs weniger als 30 Minuten dauern. Der Klinikaufenthalt ist bei einer Ringeinlage sehr kurz und beträgt bei Nachresektionen weniger als 3 Tage. Malabsorptive Verfahren verlangen in nur 1-2\% der Fälle eine Korrektur des Common Channels (Weiner 2010). Die Möglichkeit des laparoskopischen Zugangs für Revisionen nach vorgängiger offener Chirurgie ist mehrfach demonstriert worden (Weiner 2010).

\section{Zusammenfassung}

Die operative Behandlung der krankhaften Adipositas ist derzeit die einzig effektive und langfristig erfolgreiche Therapieoption. Die Ergebnisqualität hängt von einer lebenslangen optimalen Nachbetreuung ab. Die Auswahl der Operations- 
verfahren erfolgt individuell. Die Hauptprinzipien der Operationen, die heute ausschließlich minimal-invasiv ausgeführt werden, bestehen in Restriktion und Malabsorption von Fett oder in einer Kombination. Die umfangreichen Effekte auf das metabolische Syndrom haben zu einer Erweiterung der Indikation geführt. Steht die Stoffwechselerkrankung im Vordergrund, dann spricht man von metabolischer Chirurgie.

\section{Literatur}

Adams TD, Gress RE, Smith SC, Halverson RC, Simper SC, Rosamond WD, Lamonte M), Stroup AM, Hunt SC (2007) Long-term mortality after gastric bypass surgery. N Engl I Med 357, 753-761

Andersen T, Backer OG, Stokholm KH, Quaade F (1984) Randomized trial of diet and gastroplasty compared with diet alone in morbid obesity. N Engl I Med 310, 352-356

Berg T, Zurmeyer EL, Ranneberg T, Schönleben K (2001) Surgical therapy of morbid obesity using an adjustable gastric band. Report of experiences over $2 \frac{1}{2}$ years with 71 patients Med Klin (Munich) 96, 191-195

Buchwald H, Williams SE (2004) Bariatric surgery worldwide 2003. Obes Surg 14, 1157-1164

Chapman AE, Kiroff G, Game P, Foster B, O’Brien P, Ham I, Maddern G) (2004) Laparoscopic adjustable gastric banding in the treatment of obesity: a systematic literature review. Surgery 135 , 326-351

Christou N, Sampalis J, Liberman M, Look D, Auger S, McLean AP, MacLean LD (2004) Surgery Decreases Long-term Motarlity, Morbidity and Health Care use in Morbidly obese Patients. Ann Surg 240, 416-424

Dargent I (1999) Laparoscopic adjustable gastric banding: lessons from the first 500 patients in a single institution. Obes Surg 9 , 446-452

Deutsche Gesellschaft für Allgemein- und Viszeralchirurgie (DGAV) (2010) S3-Leitlinie Chirurgie der Adipositas, http://www.awmf. org/uploads/tx_szleitlinien/088-001l_S3_Chirurgie_der_ Adipositas_2010-06.pdf, abgerufen am 01.11.2011

Dindo D, Muller MK, Weber M, Clavien PA (2003) Obesity in general elective surgery. Lancet 361, 2032-2035

de Freitas Carvalho DA, Valezi AC, de Brito EM, de Souza JC, Masson AC, Matsuo T (2006) Rhabdomyolysis after Bariatric Surgery. Obes Surg 16, 740-744

Gagner M, Gentileschi P, de Csepel I, Kini S, Patterson E, Inabnet WB, Herron D, Pomp A (2002) Laparoscopic reoperative bariatric surgery: experience from 27 consecutive patients. Obes Surg 12, 254-260

Gustavsson S, Westling A (2002) Laparoscopic adjustable gastric banding: complications and side effects responsible for the poor long-term outcome. Semin Laparosc Surg 9, 115-124

Helmert U, Strube H (2004) Die Entwicklung der Adipositas in Deutschland im Zeitraum 1985-2002. Gesundheitswesen 66, 409-415

Hess DS, Hess DW (1998) Biliopancreatic diversion with a duodenal switch. Obes Surg 8, 267-282

Holeczy P, Novak P, Kralova A (2001) 30\% complications with adjustable gastric banding: what did we do wrong? Obes Surg 11, 748-751

Kurth BM, Schaffrath-Rosario A (2007) Die Verbreitung von Übergewicht und Adipositas bei Kindern und Jugendlichen in Deutsch- land. Ergebnisse des bundesweiten Kinder- und Jugendgesundheitssurveys (KiGGS). Bundesgesundheitsbl-GesundheitsforschGesundheitsschutz 50, 736-743

Larrad-jiménez A, Díaz-Guerra CS, de Cuadros Borrajo P, Lesmes IB, Esteban BM (2007) Short-, mid- and long-term results of Larrad biliopancreatic diversion. Obes Surg 17, 202-210

Lujan JA, Hernandez Q, Liron R, Cuenca JR, Valero G, Parrilla P (2004) Laparoscopic versus open gastric bypass in the treatment of morbid obesity: a randomized prospective study. Ann Surg 239, 433-437

Mensink GBM, Lampert T, Bergmann E (2005) Übergewicht und Adipositas in Deutschland 1984-2003. Bundesgesundheitsbl - Gesundheitsforsch Gesundheitsschutz 48, 1348-1356

National Institutes of Health Consensus Development Conference (1992) Gastrointestinal surgery for severe obesity. Consens Statement 9, 1-20

Prugger C, Keil U (2007) Entwicklung der Adipositas in Deutschland Größenordnung, Determinanten und Perspektiven. DMW 132, 892-897

Rubino F, Gagner M (2002) Potential of surgery for curing type $2 \mathrm{di}$ abetes mellitus. Ann Surg 236, 554-559

Schauer PR, Ikramuddin S (2001) Laparoscopic surgery for morbid obesity. Surg Clin North Am 81, 1145-1179

Sharma AM (2004) Bariatric medicine without surgery is like nephrology without dialysis. Obes Surg 14, 1145-1147

Sjöström L, Lindroos AK, Peltonen M, Torgerson I, Bouchard C, Carlsson B, Dahlgren S, Larsson B, Narbro K, Sjöström CD, Sullivan M, Wedel H; Swedish Obese Subjects Study Scientific Group (2004) Lifestyle, diabetes, and cardiovascular risk factors 10 years after bariatric surgery. N Engl I Med 351, 2683-2693

Sjöström L, Narbro K, Sjöström CD, Karason K, Larsson B, Wedel H, Lystig T, Sullivan M, Bouchard C, Carlsson B, Bengtsson C, Dahlgren S, Gummesson A, Jacobson P, Karlsson I, Lindroos AK, Lönroth H, Näslund I, Olbers T, Stenlöf K, Torgerson I, Agren G, Carlsson LM; Swedish Obese Subjects Study (2007) Effects of bariatric surgery on mortality in Swedish obese subjects. N Engl I Med 357, 741-752

Strain GW, Gagner M, Pomp A, Dakin G, Inabnet WB, Hsieh J, Heacock L, Christos P (2009) Comparison of weight loss and body composition changes with four surgical procedures. Surg Obes Relat Dis 5, 582-587

Suter M, Bettschart V, Giusti V, Heraief E, Jayet A (2000) A 3-year experience with laparoscopic gastric banding for obesity. Surg Endosc 14, 532-536

Torres IC (1991) Why I prefer gastric bypass distal roux-en-y gastroileostomy. Obes Surg 1, 189-194

Weiner RA (2010) Adipositaschirurgie. Elsevier, Amsterdam

Weiner RA, Blanco-Engert R, Weiner S, Pomhoff I, Schramm M (2004) Laparoscopic biliopancreatic diversion with duodenal switch: Three different duodeno-ileal anastomotic techniques and initial experience. Obes Surg 14, 334-340

Weiner RA, Weiner S, Pomhoff I, Jacobi C, Makarewicz W, Weigand G (2008) Laparoscopic Sleeve Gastrectomy - Influence of Sleeve Size and Resected Gastric Volume. Obes Surg 17, 32-36

Weiner S, Weiner RA, Rosenthal A, Pomhoff I (2007) Lebensqualität nach Adipositaschirurgie: Ergebnisse nach verschiedenen 0perationsverfahren - erste Ergebnisse einer prospektiven Längsschnittstudie. Chir Gastroenterol 23 (Suppl. 1), 52-54 
Westling A, Ohrvall M, Gustavsson S (2002) Roux-en-Y gastric bypass after previous unsuccessful gastric restrictive surgery. I Gastrointest Surg 6, 206-211
WHO (2000) Obesity: preventing and managing the global epidemic. Report of a WHO consultation. World Health Organ Tech Rep Ser 894:I-XII, 1-253

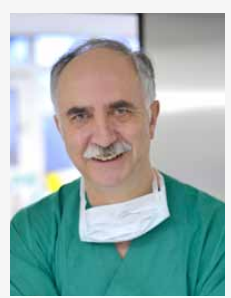

Prof. Dr. med. Rudolf Alfred Weiner

1976 Staatsexamen Universität Leipzig, anschließend Promotion zum Dr. med. an der Charité Berlin. Arzt im Kreiskrankenhaus Zschopau/Erzgebirge, 1981 Facharzt für Urologie, Klinikum „St. Georg“ Leipzig, 1984 Facharzt für Chirurgie und 1989 Oberarzt für Chirurgie am gleichen Klinikum, 1992 Habilitation an der Universität Leipzig. Ab 1993 Oberarzt für Chirurgie am Krankenhaus Nordwest in Frankfurt am Main, 1994 Privatdozent für Chirurgie an der Universität Frankfurt am Main, 1995 Subspezialisierung Viszeralchirurgie am Krankenhaus Nordwest, ab 2000 Professor für Chirurgie an der Universität in Frankfurt am Main. Seit 2001 Chefarzt für Chirurgie am Krankenhaus Sachsenhausen in Frankfurt am Main. 